\title{
A Summary of the Analysis of Some Data from Two ABI Trials
}

\author{
$\underline{\text { E. El-Mahassni }}^{\mathrm{a}}$ and G. Burgess $^{\mathrm{a}}$ \\ ${ }^{a}$ National Security and ISR Division,Defence Science and Technology Group \\ Email: Edwin.El-Mahassni@dst.defence.gov.au
}

\begin{abstract}
Data fusion can be defined as the process of integrating data and information, typically from multiple sources and source types. The key to good data fusion is the ability to utilise all available information regardless of where it comes from in order to better understand the domain under surveillance. In addition, a growing area of interest in intelligence is in Activity Based Intelligence (ABI). It requires the combination of different pieces of data, from different sources and different in their semantic nature. That is, some data might be numerical and other data might be qualitative. In order to conduct ABI, consideration must also be given to human activity and relationships as these are not typically covered in low-level intelligence gathering.

The aim of this paper is to give an overview of some of the issues and challenges involved arising from the analysis of data from two ABI trials, Pimento and Jalapeño conducted by DST Group, including integrating different levels of fusion, which involves the human element of relationships. In Pimento, the goal was to develop and formulate some concepts that could be useful in understanding ABI. Taking the GPS as a proxy for tracks, techniques were developed to derive kinematics like speed and interactions like potential meetings, and cases where two or more entities might have travelled together were derived. More complex procedures involved the use of open source software tools to better understand the locations entities have crossed or visited. Interactions were also visualized through social network tools. In Jalapeño, many of these concepts remained with the aim there to combine data from different sources so that not only basic kinematic information was provided ("What") but also some contextual ("Why") data could be gleaned.
\end{abstract}

Keywords: Activity-based intelligence, data fusion, social networks 


\section{INTRODUCTION}

For people working in the geospatial domain, there is often the need to combine diverse data sets. Data fusion can be defined as the process of integrating data and information, typically from multiple sources and source types. However, this is not trivial as information may contain uncertainty or imprecision. In addition, the data from these sources might need to be refined or transformed so that a normative or standardised scale is used when combining different pieces of information. That is, sensor data and eyewitness accounts might be available, but the former is precisely measured whilst the latter provides context and is more qualitative. A further issue in intelligence is the need to update new data being observed. These tasks can be non-trivial, but the key to good data fusion is the ability to utilise all available information regardless of where it comes from in order to better understand the domain under surveillance. Correspondingly, a growing area of interest in intelligence is in Activity Based Intelligence (ABI). It requires the combination of different pieces of data, from different sources and different in their semantic nature. In order to conduct ABI, consideration must also be given to human activity and relationships, which are not typically covered in low-level intelligence gathering. The aim of this paper is to give an overview of some of the issues and challenges involved arising from the analysis of data from two ABI trials, conducted by DST Group, including integrating different levels of fusion, which involves the human element of relationships (OPSEC, 1996).

\section{ACTIVITY-BASED INTELLIGENCE}

Intelligence can be defined as the evaluation resulting from the collection, collation, evaluation, analysis, integration, and interpretation of collected information (OPSEC, 1996). A function of intelligence is to reduce ambiguity or the uncertainty inherent in the interpretation of observed activities. Intelligence can be divided into two categories: strategic and operational. Strategic intelligence is long-term and often focused on policy to address the national interest. This area covers politics, military affairs, economics, social interactions and technological developments. Operational intelligence is concerned with near-term or current events and is often used to determine the capability and feasibility of a current program or operation.

Activity-Based Intelligence (ABI) is defined by the Office of the Undersecretary of Defense in the US as "a discipline of intelligence where the analysis and subsequent collection is focused on the activity and transactions associated with an entity, a population, or an area of interest" (Phillips, 2012). ABI integrates multiple sources of intelligence, requiring kinematic and qualitative data. That paper also notes the presence, activities, culture, social structure/organization, networks and relationships, motivation, intent, vulnerabilities, and capabilities of humans (either individually or in groups) across all domains of the operational environment are a vital and integral part of ABI. In ABI, analysts seek to discover abnormality in the observations, trends and patterns by correlating multiple sources of information in space and time (BAE, 2013).

ABI's aims can be summarised as follow (Phillips, 2012):

1. Characterize and locate activities and transactions.

2. Identify and locate entities conducting the activities and transactions.

3. Identify and locate networks of actors. Meetings or an entity following another entity lead to the inference there is a relationship between two entities.

4. Understand the relationships between these networks.

5. Develop patterns of life which allow detection of anomalous behaviour or changes in patterns.

Miller (Miller, 2013) makes a similar observation. He quotes Gauthier from NGA (National GeospatialIntelligence Agency) who in GEOINT2012 stated ABI is "a rich new data source for observing the world and the connectedness between objects and entities in the world."

These definitions underline the nebulous nature of ABI, its capability, and relative infancy. ABI consists of multiple layers of information which often come from different types of sources. This means that ABI analysis is more than tracking and following a target of interest with a particular pre-defined goal.

With that in mind, Pimento was run as the inaugural trial under the Wide Area Surveillance and Activity Based Intelligence (WASABI) project in May 2014. The goal of this trial was to collect data from a variety of sources, including an airborne sensor platform with the data then being analysed. From late November until early December of that year, the Jalapeño trial was run also as part of project WASABI. This paper provides a 
summary of the techniques developed and the analysis of data obtained in both trials as well as an overview of the issues and challenges arising from the analysis of this trial data - including in integrating different levels of fusion and involving the human element of relationships.

\section{PIMENTO TRIAL}

This trial sought to address three objectives:

1. To develop and discuss concepts for tools that would assist an intelligence analyst to infer activities conducted during the May 2014 trial and during similar operations.

2. To establish descriptions for the actual activities conducted during the May 2014 trial. These may include statements like "Person A was walking towards the shops" or a higher level description like "This group of people are organising for a rally with the intent to disrupt the flow of traffic." These descriptions may form the basis for assessing tools which would assist an intelligence analyst.

3. To provide suggestions about intelligence aspects of subsequent trials, such as how to infer more about the activities, social relationships and intents.

\subsection{GROUND ACTIVITIES}

The notional mission for the plane operators was to conduct wide area surveillance of a pre-specified region and to detect and monitor anomalous activities or activities of interest such as a group of people planning to cause a public nuisance. The aim of deploying airborne sensors was therefore to provide persistent coverage of a large area with a wide-area, low-resolution camera to compliment a narrow area, high-resolution camera. Lasch described a similar concept (4) and writes: "Two commonly used forms of motion imagery in the National Security Domain include Full Motion Video (FMV) and Wide Area Motion Imagery (WAMI). Each has unique characteristics and applicability to ABI analysis." He goes on to say: "FMV is typically high frame-rate (>20fps), relatively narrow field of view imagery with high frame-to-frame overlap...FMV is widely available for intelligence analysis from collection platforms". For WAMI, he also notes "(it) is typically low framerate $(<20 \mathrm{fps})$, wide field of view motion imagery used in persistent surveillance applications. Generally, the information gathered from these sensors is used in real time to conduct forensic analysis." Both types of sensors compliment each other, with FMV "well-suited to derive detailed activity intelligence for features of specific targets", while WAMI sensors "give ABI analysts broader coverage although at a lower frame rate and at often lower spatial resolution than comparable FMV sensors."

Fourteen volunteers from DST Group conducted the Ground Activity during part of the Pimento trial. The personnel were split into two teams, that undertook different ground activities for a period of about 90 minutes. The activity was designed so there would be two teams conducting simultaneous activities that at the surface might either be innocent or suspicious. SNA's aims were to see if by looking at the patterns of interactions between tracks, an analyst could determine: the presence and composition of teams, the structure of each team and the team missions. For instance, it might not be possible to discern what the team missions were, but determining which are the two distinct teams might be more achievable.

The first team was assigned the role of Delinquents. The Delinquent Team was tasked with simulating the actions of a group of people intent on creating or organizing a disturbance. The second team was called the Treasure Hunters. It was given a list of locations to visit and photograph. Some were described using geospatial coordinates on a map, while others were conveyed using a custom-made reverse-geocaching device.The original aim was for the Treasure Hunters to perform a set of activities that were "benign" compared to the Delinquents.

\subsection{DATA COLLECTION}

Data from different sources were collected, primarily for post-trial analysis. Additional potential useful information for analysis included simulated messages exchanged by participants. Smartphones were also purely used here to aid the participants in carrying out the ground activities.

The types of motion undertaken by participants and the interactions between participants were identified using track data. The motion types and interaction types are described in Section 3.3.

Using the track data, analysis of the types of motion undertaken by participants (such as walking, running, driving or standing still), and identification of different types of interactions between individuals (such as 
meeting, walking together, driving together or following each other in separate vehicles) were undertaken. Identifying interactions between individuals and geographic locations, like loitering in a carpark, visiting a particular shop, or stopping at a train station can also be performed.

\subsection{SOCIAL INTERACTIONS}

This section discusses the interactions of the entities in relation to their immediate environment and to other entities. Options for processing WASABI data to support an intelligence analyst are provided. This section also describes the nature of the data used before processing track (or equivalent) information to obtain locations of interest and motion of entities. Algorithms for producing descriptions of events such as meetings between entities are outlined.

TRACK DATA. It is assumed the track data is accurate in relation to the geospatial database features so reasonable associations can be made. Where the track data are sparse and/or uncertain and the entity is a vehicle, it is possible to use a road network to infer the route, even with the identification of ambiguities present (7). For the Pimento trial, the track data were obtained via GPS (through devices carried by participants) or video (from airborne platform).

MOTION BEHAVIOUR CLASSIFICATION. The Pimento trial objectives included performing Activity Based Intelligence, utilising Wide Area Surveillance assets, based on the scripting scenario mentioned earlier. Airborne cameras provided imagery of regions and entities of interest and conceptually, the image data was processed to form and maintain tracks on entities of interest. Then, processing was performed so that the track data was classified to provide entity descriptions which would support an intelligence analyst. The GPS data was considered as a proxy for track data based on imagery from the camera systems.

Data Association was trivial for GPS data but is typically complex for detections arising from video data. While video data may contain attribute information such as colour of vehicle/clothing, this information is absent in the GPS data. The trial started around noon on a clear sunny day and ended just over 1.5 hours later. There were six GPS "tracks" recorded, henceforth denoted as $A, B, \ldots, F$.

To assist the analyst to interpret the entity data, the entity motion was classified into one of the following: i) Stopped, ii) Walking, and iii) Using motorised transport.
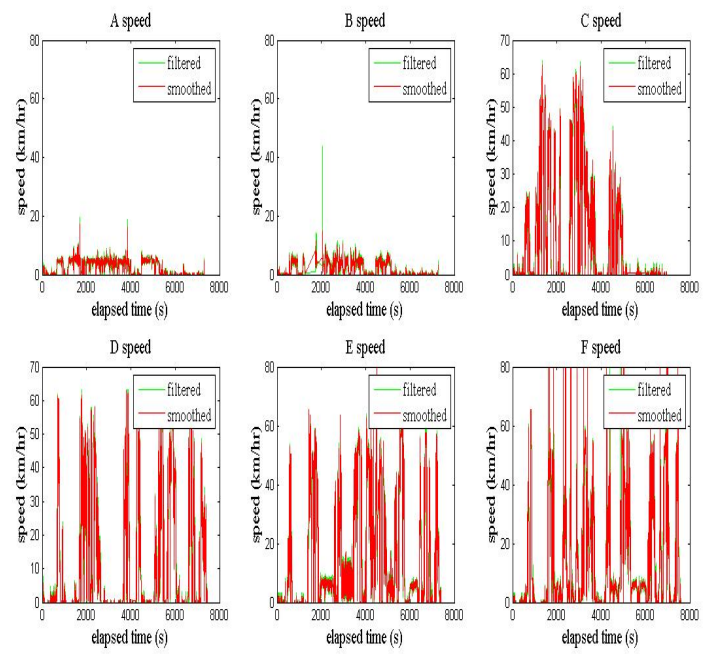

Figure 1. Speed Recorded by Each Entity per Minute Interval
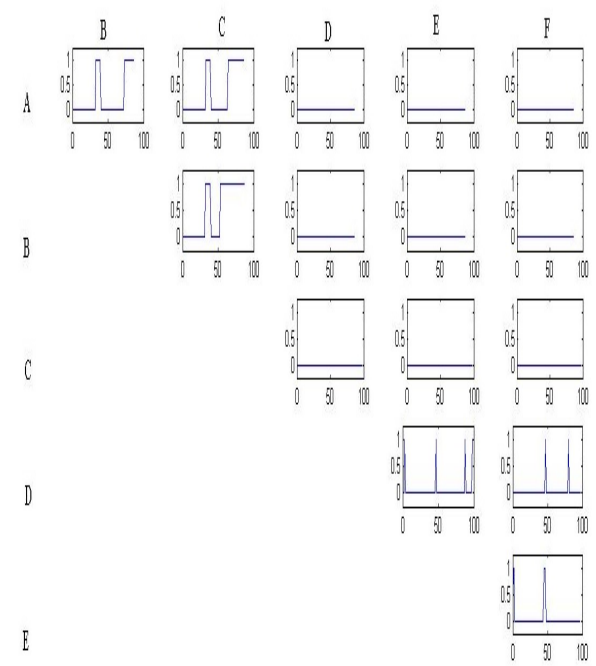

Figure 2. Plots of "meetings" between any two entities

From Figure 1, all entities except $A$ and $B$ have taken some form of motorised transport. However, there were some problems with the data. Given that no car is believed to have travelled over $80 \mathrm{~km} / \mathrm{hr}$ (and even far less likely that it would be over $200 \mathrm{~km} / \mathrm{hr}$ as some data points for track F indicated), the plots were maximally bound to that speed. The reason for this is not precisely known, but it could be that the GPS signal bounced off the buildings nearby.

Interactions between individuals are either directly or indirectly observed. These may be from individuals seen in the same location and at the same time, or individuals linked by a sequence of related activities. Entities 
have certain attributes (e.g. ID, position, associated car or train) linking them to other entities. Determining the presence of entity interactions could be considered merely a subset of a full list of possible events. However, in ABI, social ties and networks are crucial, it could be argued they should be emphasized. There are two types of descriptions an entity could be categorised under: their events or tasks carried out and their social interactions. These are now described by classifying the pair-wise entity interactions into one of the following: i) Crossed paths, ii) Met each other, iii) Travelled together, and iv) Were not close.

To determine whether an interaction between two entities took place, three key measurements were considered: the distance between them, the duration of a suspected interaction and the entities' velocities at that time.

Using the "met" rule, the likelihood of meetings occurring can be derived as shown in Figure 2.A, B and C met at around 35 minutes after the exercise started for about 10 minutes and D, E and F met for about 5 minutes around the 45 minute mark. D also then meets up with $\mathrm{F}$ and a short time later and on a separate occasion with E. The observed interactions identify two distinct groups emerge: namely A, B, C and D, E, F.

SOCIAL NETWORK ANALYSIS (SNA). Social network analysis represents relationships between social entities, events, locations and other objects in the system of interest as a network. Entities are represented as nodes, and edges represent relationships between the entities such as kinship relations, communication events, place of birth and other persistent or ephemeral relations of interest. SNA combines social theory, mathematical (statistical and graph theoretic) analysis and visualisation (Borg, 2013), (Scott, 2012) and (Wasserman, 1994). An analyst can make assessments about individuals and various aspects of the network that may not be immediately obvious, like, who is the leader, who is most likely to take over if the leader is removed, who else is in a position of influence, what gives people influence in this network, who is likely to have access to useful information, how difficult would it be to disrupt this network, and even, are there parts of this network that cannot be seen. The entity interactions identified can then be converted into a network representation that can potentially be analysed using SNA methods.

\subsection{GEOSPATIAL EVENTS}

This section describes the recognition of geospatial events derived from the combination of navigation data with a geospatial database and their potential use in higher level data fusion processing.

Events such as entering a shop or following a particular street can be used to summarise the actor's journey in a manner that can be understood by a human operator or algorithms that look for correlations between the motions of actors in a group. Such a summary can also be interpreted as a series of state transitions, allowing behaviour to be represented as a matrix indexed by the current state versus the next state. Given an entity's location, this approach permits an individual's behaviour to be predicted by choosing the highest probability state transition. It also allows anomalous behaviour to be detected, either in the context of the entire population or relative to the actor's normal behaviour.

GEOSPATIAL DATABASE. The community project database OpenStreetMap (OSM) (Openstreetmap, 2012) could also be used in this work. It provides free geospatial data to anyone. Data are contributed by participating users and can be used for a multitude of applications. In addition to map imagery, the service allows access to the underlying database, which includes features such as streets, buildings, political and geographic boundaries, rivers, shops and amenities including park benches and ATMs.

Given the crowd sourced nature of this service, coverage of the database is variable, somewhat inconsistent, and changes over time. However, it is valuable for exploring the potential for translating navigational data into geospatial events. OSM data could be stored using a number of different data structures, including a list of nodes and their corresponding locations; a list of "ways" with their corresponding sequence of nodes and descriptive attributes; and a list of entities such as amenities with locations and descriptions.

ASSOCIATING TRACK DATA WITH OBJECTS IN A GEOSPATIAL DATABASE. OSM data could be stored using a number of different data structures, including a list of nodes and their corresponding locations; a list of "ways" with their corresponding sequence of nodes and descriptive attributes; and a list of entities such as amenities with locations and descriptions.

Buildings and streets could be coded using ways. In the case of streets, the sequence of nodes corresponds to the middle of the road at locations where there are intersections or changes in direction. For a building, the sequence of nodes corresponds to the building's outline. Most streets have names, although roundabouts and many linking roads are not named. Buildings may or may not have names and also in this instance the track 
data does not include height which would not give the precise location. Where a building contains a shop, the building may take on the name of the shop, or it may be necessary to search the list of entities to find those that fall within the building of interest. Where more than one shop exists within a building, the one of interest may be the one closest to the navigation data point under test.

OSM data could be parsed to Matlab (Filippidis, 2012). To find if the navigation data point was associated with a street, a rectangle was generated for each adjacent pair of nodes in its "way". This rectangle had a length that exceeded the length of the road segment so that the navigation data would be associated with the street even if it passed through an adjacent intersection, and a width sufficient to capture any adjacent footpath. (Some footpaths are listed explicitly in the database, but these were not used.) A test was then made to see if the data point fell within the resulting rectangle.

EVENT PROCESSING. A collection of event sequences can be used to form a model of normal behaviour. Such a model may be used for prediction and anomaly detection. A simple approach is to generate a matrix that represents the transitions from one event to another. This matrix is formed with rows representing the current event and columns the next event. The entries represent the probability of the state transition, i.e. It is a Markov chain. So that the prior distribution is uniform and has a high level of uncertainty, the matrix is initially filled with ones.

\section{JALAPEÑO TRIAL}

Conducted on late November and early December of 2014, this trial shared many of the same features as Pimento , but there were also new aspects which are worth mentioning.

1. In this trial, video data was used in the analysis.

2. Artificial social media data were also created by participants and used to assist analysis.

3. While the trial scenario was the same as that in Pimento, the ground activities were subtly different. Instead of having two d istinct groups doing sets of activites, there was now one group carrying out all activities involving the transfer and movement of a package (which contained a GPS device).

\subsection{GROUND ACTIVITIES}

As in Pimento, this team consisted of DST Group volunteers who set out to carry a set of prescribed activities. Unlike the Pimento trial, there was one group (instead of two) working together and cohesively. Here, the task was to coordinate a public nuisance event in the form of a protest. The data collection for this trial is similar to Pimento. However, in this exercise, there was also the injection of artificial social media information as another source of data which could be exploited. One of the objectives of this exercise was to determine which GPS track is inside the package and understand its movements.

\subsection{DATA ANALYSIS}

Just like in Pimento, the GPS tracks are analysed so that interactions among them may be detected. A log with all the potential interactions with latitude and longitude values is generated. These interactions are then cross-checked with the video data for verification. There were some moments where the video data could not determine whether there was an interaction or not. As an example, there was an instance where the camera's view was obscured. In this situation, social media data can be examined with a simple key word search to detect not only potential interactions but also to provide some context on the reason for the interaction. The importance for fusing "hard" and "soft" information cannot be understated. Llinas and Scrofani write "The experiences of Iraq and Afghanistan have also shown that inputs and supporting information (such as contextual information, either real-time or in static databases) of a textual, linguistic type have proven helpful if not critical toward yielding improved intelligence analysis capabilities. This type of information has come to be called "soft", to distinguish it from "hard, physics-based" data provided by the usual repertoire of ISR sensing devices across the electromagnetic spectrum." (Llinas, 2014).

Finally, recall that the parcel in question contained a GPS unit. If one assumes that this parcel was handled by most of the other entities, then it would be expected to be the most connected entity. Displaying all the interactions in the form of a social network shows that entity B is the parcel (Figure 3). 


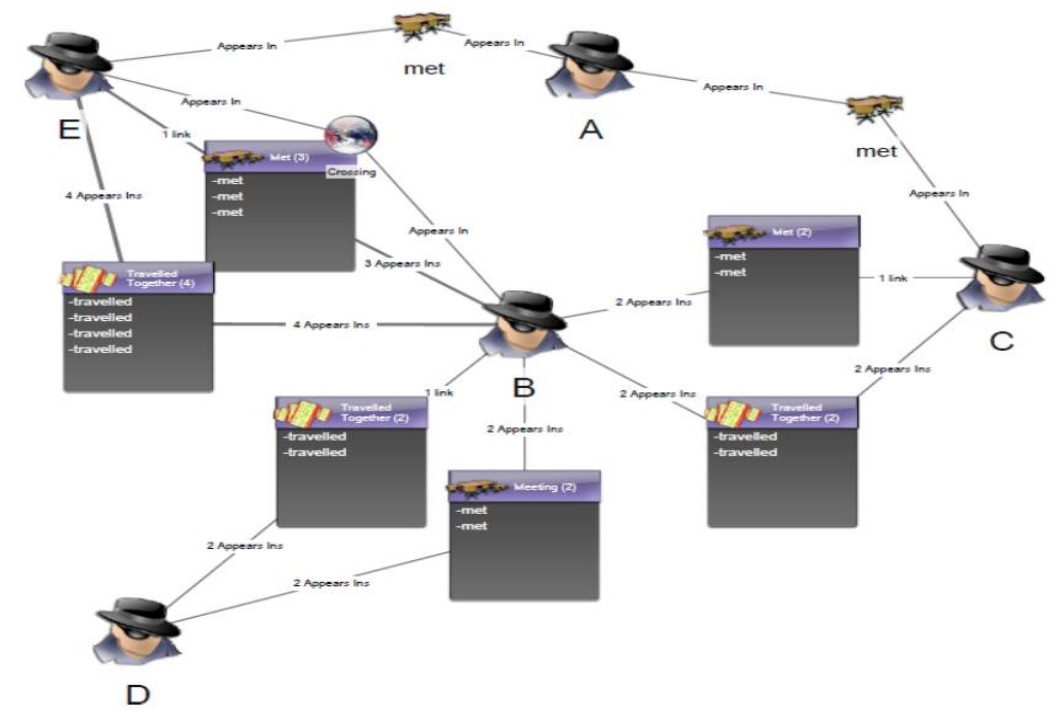

Figure 3. Graphical representation of entities and their interactions

\section{CONCLUSION}

This paper provided an overview of recent ABI trials. In Pimento, the aim was to develop and formulate concepts that could be useful in understanding ABI. In Jalapeño, these concepts remained with the aim there to combine data from different sources to obtain both kinematic information ("What") and contextual ("Why") data. The integration of numerical with qualitative data continues to be an ongoing and challenging problem.

\section{REFERENCES}

BAE (2013). Watching Your Actions: Finding the Right Hay in a Haystack. BAE Systems Magazine.

Borgatti, S. P., Everett, M. G. and Johnson, J. C. (2013). Analyzing Social Networks. SAGE Publications.

Filippidis, I. (2012). Openstreetmap functions. http://www.mathworks.com.au/matlabcentra/fileexchange/35819-openstreetmap-function.

Lash, T. (2013). Uses of Motion Imagery in Activity-Based Intelligence. Proc. of SPIE, 8740, 1-7.

Llinas, J. and Scrofani, J. (2014). Foundational Technologies for Activity-Based Intelligence - A Review of the Literature. Naval Postgrad School Tech. Report NPS-EC-14-001.

Miller, G. (2013). Activity-Based Intelligence Uses Metadata to map Adversary Networks. Defence News, C4ISR Digital Edition.

Morelande, M., Duckham, M., Kealy, A. and Legg, J. (2015). Bayesian path estimation using the spatial attributes of a road network, Proc. ICASSP 2015.

OPSEC Support Staff. (1996). Intelligence Threat Handbook. DIANE Publications. Co., Ch2.

OpenStreetMap (2012) http://www.openstreetmap.org.

Phillips, M. (2012). A Brief Overview of ABI and Human Domain Analytics. Trajectory magazine, NGA case number 12-463, Sept. 28, 2012. Online: http://trajectorymagazine.com/web-exclusives/item/1369human-domain-analytics.html.

Scott, J. (2012). Social Network Analysis. SAGE Publications.

Wasserman, S. and Faust, K. (1994). Social Network Analysis: Methods and Applications. Cambridge University Press. 